\title{
Flexible Sigmoidoscopy: Optimal bowel preparation with enema versus polyethylene glycol
}

\section{Ghimire $\mathbf{R}^{1}$, Limbu $\mathrm{Y}^{2}$, Chakradhar $\mathbf{R}^{2}$}

${ }^{1}$ Roshan Ghimire, Assistant Professor; ${ }^{2}$ Yugal Limbu; ${ }^{2}$ Rupesh Chakradhar, MS Resident; Department of Surgery, Kathmandu Medical College Teaching Hospital, Sinamangal, Kathmandu.

\begin{abstract}
Background: Lower gastrointestinal endoscopy requires adequate bowel preparation. However there is no unanimous recommendation on choice of agent of bowel preparation for the purpose of screening flexible sigmoidoscopy.

Objective: To compare the quality of bowel preparation and patient's tolerance between Enema (Solution of Glycerin $15 \% \mathrm{w} / \mathrm{v}$ and Sodium Chloride 15\%w/v)and Polyethylene glycol for screening flexible sigmoidoscopy.

Methodology: All consecutive patients undergoing screening flexible sigmoidoscopy were included in the study. We prospectively divided the patients into two groups. Group A received per rectal enema and group B received oral bowel preparation with polyethylene glycol. Adequacy of bowel preparation and patient's tolerance between two groups was graded. Data were analysed using Microsoft Excel 2010.

Results: Results were comparable regarding quality of bowel preparation between two groups ( $\mathrm{A}$ and B), excellent in $20 \%$ and $33.33 \%$ and good in $60 \%$ and $50 \%$ respectively. Almost all patients could tolerate the preparation agent in Group A ( $n=28$ ). In-group B, eight patients(26.66\%) considered the oral preparation agent difficult to tolerate and two patients $(6 \%)$ were unable to complete the preparation.

Conclusion: Enema(Solution of Glycerin $15 \%$ w/v and Sodium Chloride 15\%w/v) andPolyethylene glycol have similar quality of bowel preparation and for screening sigmoidoscopy. Enema is adequate and much easily tolerable compared to polyethylene glycol.
\end{abstract}

Key words: Adequecy; Flexible Sigmoidoscopy; Preparation; Screening; Tolerance

\section{INTRODUCTION}

T he gastrointestinal tract is susceptible to numerous pathologies such as congenital, inflammatory, infectious, and neoplastic lesions. Sigmoidoscopy is used frequently these days as a diagnostic and screening tool for symptomatic colorectal diseases ${ }^{1}$. Diagnosis of colorectal diseases is facilitated by endoscopic procedure which also enables to take biopsy for histological evaluation ${ }^{2}$. To assess the lower gastrointestinal tract via endoscopy, adequate bowel preparation is an essential prerequisite $^{3}$. Thus it is necessitous indispensible to determine which agent gives the best quality of bowel preparation with the least effects and hindrance to the patients.

\section{Address for correspondence}

Dr. Roshan Ghimire,

Assistant Professor; Department. of Surgery,

Kathmandu Medical College Teaching Hospital, Sinamangal

E-mail: roshan194@hotmail.com
Good quality and easy tolerability are the main desirable elements of bowel preparation. A Study by Yusuf A et al. concluded oral preparation had significantly better bowel preparation compared to phosphate enema4. However study done by Preston et al. concluded that a single enema was adequate for bowel preparation ${ }^{3}$.

As the completeness of sigmoidoscopy is highly dependent on the quality of bowel preparation, the agent selected for this purpose is of great value. Moreover, patient tolerance and cost effectiveness should also be taken into consideration when choosing an agent for bowel preparation.

We conducted this study, comparing enema Solution of Glycerin 15\% w/v and Sodium Chloride 15\%w/v $30 \mathrm{ml}$ versus oral polyethylene glycol (PEG) for bowel preparation in flexible sigmoidoscopy on the basis of adequacy and overall patient's tolerance. 


\section{METHODOLOGY}

Ethical clearance for this study was obtained from the Institutional Review Board of Kathmandu Medical College Teaching Hospital. After written consent was obtained, 60 consecutive patients scheduled for routine screening flexible sigmoidoscopy were enrolled in the study. Patients were alternatively allocated to group $A$ and group B. Patients in group A received per rectal enema one-hour prior to the procedure whereas 30 patients in group B were prepared with 1-2 litres of oral preparation of peglec ${ }^{\mathrm{TM}} 118 \mathrm{gm}$ four to six hours prior to the procedure. A single endoscopist performed the procedure over a period of three months (15 January 2015 to $15^{\text {th }}$ April 2015).

\section{INCLUSION CRITERIA:}

- Consecutive male and non-pregnant female patients more than 18 years of age who required screening sigmoidoscopy.

- Those who gave consent.

\section{EXCLUSION CRITERIA:}

- Previous colorectal surgery

- Patients with clear indications of colonoscopy

- Medical conditions with reduced renal functions that could increase the risk of receiving oral preparations

- Those who did not give consent

Method of administration of the enema: Patient was asked to lie on the left lateral position with knee bent and arm resting comfortably. The enema plastic bottle tip after lubricating with lidocaine jelly was gently inserted into the rectum through the anus after which the bottle was emptied into the rectum. The patient was advised to retain the enema until the urge of defecation or for $10 \mathrm{~min}$ whichever came first.

Content of enema: Solution of Glycerin 15\% w/v and Sodium Chloride 15\% w/v $30 \mathrm{ml}$

Method of administration of oral preparation: On the morning of the procedure, patient was advised to drink oral preparation of polyethylene glycol made in one to two liters of water. To prevent electrolyte imbalance and avoid dehydration, oral rehydration solution was given with the preparation. After four to six hours of intake of oral preparation, procedure was done.

Content of preparation: Poly-ethylene glycol $118 \mathrm{gm}$ in 1-2 litres of water, with electrolyte supplementation.
The endoscopist rated bowel preparation quality in four groups i.e. excellent, good, fair and poor as per predetermined criteria based on article published by Schanz et $\mathrm{al}^{5}$.

- $\quad$ Poor = Unprepared colon segment with mucosa not seen due to solid stool that cannot be cleared.

- Fair = Portion of mucosa of the colon segment seen, but other areas of the colon segment not well seen due to staining, residual stool and/or opaque liquid.

- Good = Minor amount of residual staining, small fragments of stool and/or opaque liquid, but mucosa of colon segment seen well.

- Excellent $=$ Entire mucosa of colon segment seen well with no residual staining, small fragments of stool or opaque liquid. The wording of the scale was finalized after incorporating feedback from three colleagues experienced in colonoscopy.

Patients were asked to rate how difficult it was for them to administer the bowel preparation agents (oral and enema) as: easy, tolerable, difficult or intolerable.

\section{RESULTS}

Total number of patients enrolled in the study was 60,30 in each group. Mean age of patients in group $A$ and group $B$ was 47.5 years and 49.3 years respectively (table 1 )

\section{Table 1: Patient Characteristics}

\begin{tabular}{lcc|}
\hline & Group A & Group B \\
\hline Male & 18 & 14 \\
\hline Female & 12 & 16 \\
\hline Mean Age (years) & 47.5 & 49.3 \\
\hline Male gender (\%) & 60 & 46.66 \\
\hline Mean BMI (kg/m2) & 23.33 & 22.42 \\
\hline Prior sigmoidoscopy (\%) & 11.2 & 12.4 \\
\hline
\end{tabular}

The quality of bowel preparation was excellent in $20 \%$ of the patients in group $A$ and $33.33 \%$ of the patients in group B. Similarly, it was good in $60 \%$ and $50 \%$ of patients in Group A and Group B respectively (table 2).

Most patients could tolerate the preparation agent in group A with only two patients (6.6\%) finding it difficult. In group B eight out of thirty patients (26.66\%) considered the preparation agent as difficult to tolerate and two out of thirty patients (6\%) were unable to complete the preparation (table 3). In Group A, two (6.66\%) patient required repeat bowel preparation with PEG to do full colonoscopy as per the findings of sigmoidoscopy. 
Table 2: Adequacy of bowel preparation for sigmoidoscopy

\begin{tabular}{lccccc} 
& Excellent & Good & Fair & Poor & Total \\
\hline Group A $(n=30)$ & 6 & 18 & 4 & 2 & 30 \\
\hline Group B $(n=30)$ & 10 & 15 & 4 & 1 & 30 \\
\hline
\end{tabular}

Table 3: Tolerance to bowel preparation agent (as graded by patients)

\begin{tabular}{lccccc} 
& Easy & Tolerable & Difficult & Intolerable & Total \\
\hline Group $A(n=30)$ & 15 & 13 & 2 & 0 & 30 \\
Group B $(n=30)$ & 5 & 15 & 8 & 2 & 30 \\
\hline
\end{tabular}

This data suggests that the patient compliance with enema is much higher than that of oral PEG. Mean procedure time between the groups was comparable with 12.5 min and 11 min in-group $A$ and $B$ respectively.

\section{DISCUSSION}

Flexible sigmoidoscopy is an indispensible modality of imaging technique for the diagnosis of various colorectal diseases ${ }^{6}$ and is a proven screening modality for colorectal cancer ${ }^{7-8}$. In our study we have included all patients visiting outpatient department with complain of perianal diseases requiring screening flexible sigmoidoscopy after clinical assessment.

Several studies have assessed bowel cleansing agents, such as enemas, sulfate-free polyethylene glycol electrolyte lavage solution, bisacodyl, senna, cascara, castor oil, magnesium citrate, and oral sodium phosphate ${ }^{9,10,11}$. Though the best preparation as optimal bowel cleansing regimen for screening flexible sigmoidoscopy is debatable especially regarding patient compliance ${ }^{19,20}$. In our study, enema group has better compliance (50\%) because the whole procedure was not time consuming. Oral preparation has poor tolerance because of taking one to two liters of preparation on an empty stomach (33.33\%).

The American Society for Gastrointestinal Endoscopy and study done by Weiss and Watkins recommend one or two enemas as the bowel preparation of choice for flexible sigmoidoscopy ${ }^{19,21}$. Likewise, Preston et al. ${ }^{22}$ found that one hypertonic phosphate enema one hour before flexible sigmoidoscopy was effective in which $80 \%$ of patients had an adequate or excellent preparation. In our study excellent preparation was achieved by oral preparation (33.33\%) but good preparation was comparable between two groups (60\% in enema group).

The main drawback of enema preparation is the difficulty in doing a full colonoscopy in the same sitting because of poor bowel preparation above sigmoid colon. In two patients, $6.66 \%$ of enema group required repeat oral preparation for colonoscopy because of findings in sigmoidoscopy i.e multiple polyps in sigmoid colon and proctitis. The scope accesss upto 45 from anal verge in enema group is better in terms of visibility. Similar findings were noted in a study done by Wendy S Atkin et al $^{21}$.

However, contradictory to our study, in a study by Sharma et al. ${ }^{23}$ where they compared an oral preparation consisting of magnesium citrate and 2 bisacodyl tablets the evening before flexible sigmoidoscopy with 2 hypertonic phosphate enemas administered on arrival in the endoscopy suite for screening sigmoidoscopy, they found that the oral preparation was better tolerated, resulted in a superior quality colonic preparation. Regarding cost effectiveness between two groups, definitely enema group has low cost, as oral preparation is expensive.

In our study however, the efficacy of oral PEG bowel preparation compared with enema was equivalent and with the added advantage of enema being much more easily tolerated by the patients. The only limitation of enema was difficulty in performing a complete colonoscopy when required in the same setting.

\section{CONCLUSION}

In conclusion, the effectiveness of the bowel-cleansing regimen for screening sigmoidoscopy with oral polyethylene glycol vs. enema was comparable and enema was relatively much easily tolerable.

However it should be noted that there are still conflicting data regarding the optimal agent and method for bowel preparation for screening sigmoidoscopy and further large scale study is required to ascertain the best agent for the procedure. 


\section{REFERENCES}

1. B. Joseph Elmunzer, Rodney A. Hayward, Philip S. Schoenfeld, Sameer D. Saini, Amar Deshpande, Akbar K. Waljee.(2012) Effect of Flexible SigmoidoscopyBased Screening on Incidence and Mortality of Colorectal Cancer: A Systematic Review and MetaAnalysis of Randomized Controlled Trials. PLOS Medicine 9(12):e1001352.[ DOI ]

2. Robinson Richard J, StoneMargaret, John F.Sigmoidoscopy and rectal biopsy: a survey of current UK practice. European Journal of Gastroenterology \& Hepatology: 8(2): 149-152. Feb 1996. February 1996.[ Full Text ]

3. Preston KL1, Peluso FE, Goldner F. Optimal bowel preparation for flexible sigmoidoscopy--are two enemas better than one? Gastrointest Endosc. 1994 Jul-Aug;40(4):474-6. [ PubMed ]

4. A Yusuf, S Paranahewa, P Mayhead. Comparison of low volume polyethylene glycol-electrolyte solution (PEG-ELS) and phosphate enema in flexible sigmoidoscopy: a large retrospective study. Gut 2017;66:A7. [ Full Text ]

5. S. Schanz, W. Kruis, O. Mickisch et al. Bowel preparation for colonoscopy with sodium phosphate solution versus polyethylene glycol based lavage: A multicenter trial. Diagnostic and Therapeutic endoscopy 2008; 2008: 713521[ DOI ]

6. Andreyer $H J$, Vlarianos $P$, Blake $P$, Deamaley $D$, Norman AR, Tiat D. A Gastrointestinal symptoms after pelvic radiotherapy: role for the gastroenterologist? Int J Radiat Oncol Biol Phys 2005; 62: 1464-71. [ DOI ]

7. HarrisA.Guidanceontheindications for diagnostic upper Gl endoscopy, flexible sigmoidoscopy, and colonoscopy, 2013. ( Cited on 2017/12/17). [ Full Text ]

8. Robert E. Schoen, Paul F. Pinsky, Joel L. Weissfeld, Lance A. Yokochi, Timothy Church, Adeyinka O. Laiyemo et al.Colorectal-cancer incidence and mortality with screening flexible sigmoidoscopy. N Engl J Med 2012; 366: 2345-57. [ DOI ]

9. Davis GR, Santa Ana CA, Morawski SG, Fordtran JS. Development of a lavage solution associated with minimal water and electrolyte absorption or secretion. Gastro- enterology 1980;78:991-5.[ PubMed ]

10. Clarkston WK, Tsen TN, Dies DF, Schratz L, Vaswani SK, Bjerregaard P. Oral sodium phosphate versus sulfate-free polyethylene glycol electrolyte lavage solution in outpatient preparation for colonoscopy: a prospective comparison. Gastrointest Endosc 1996 Jan; 43 (1):42-8[ PubMed]
11. Borjke B, Pedersen R, Misje Lund G, Enehaug JS, Berstad A. Effectiveness and acceptability of three bowel cleansing regi- mens. Scand J Gastroenterol 1991;26:162-6. [ PubMed ]

12. Marshall JB, Pineda JJ, Barthel JS, King PD. Prospective, randomized trial comparing sodium phosphate solution with polyethylene glycol-electrolyte lavage for colonoscopy prepa- ration. Gastrointest Endosc 1993;39:631-4. [ PubMed ]

13. Golub RW, Kerner BA, Wise WE, Meesig DM, Hartmann RF, Khanduja KS, et al. Colonoscopic bowel preparations: which one? Dis Colon Rectum 1995;38:594-9. [ DOI ]

14. Afridi SA, Barthel JS, King PD, Pineda JJ, Marshall JB. Prospective, randomized trial comparing a new sodium phos- phate-bisacodyl regimen with conventional PEG-ES lavage for outpatient colonoscopy preparation. Gastrointest Endosc 1995;41:485-9. [ DOI ]

15. Cohen SM, Wexner SD, Binderow SR, Nogueras JJ, Daniel N, Ehrenpreis ED, et al. Prospective, randomized, endoscopic- blinded trial comparing precolonoscopy bowel cleansing methods. Dis Colon Rectum 1994;37:689-96. [ PubMed ]

16. Kolts BE, Lyles WE, Achem SR, Burton L, Geller AJ, MacMath T. A comparison of the effectiveness and patient tol- erance of oral sodium phosphate, castor oil, and standard electrolyte lavage for colonoscopy or sigmoidoscopy prepara- tion. Am J Gastroenterol 1993 Aug; 88(8) : 1218-23..[ PubMed ]

17. Vanner SJ, MacDonald PH, Paterson WG, Prentice RSA, Da Costa LR, Beck IT. A randomized prospective trial comparing oral sodium phosphate with standard polyethylene glycol- based lavage solution (Golytely) in the preparation of patients for colonoscopy. Am. J Gastroenetrol 1990 April; 85 (4): 422-7[ PubMed ]

18. Henderson JM, Barnett JL, Turgeon DK, Elta GH, Behler EM, Crause I, et al. Single-day, divided-dose oral sodium phosphate laxative versus intestinal lavage as preparation for colonoscopy: efficacy and patient tolerance. Gastrointest Endosc 1995;42:23843. [ PubMed]

19. Weiss BD, Watkins S. Bowel preparation for flexible sigmoid- oscopy. J Fam Pract 1985;21:285-7. [ Full Text ]

20. Cohan CF, Kadakia SC, Kadakia AS. Serum electrolyte, min- eral, and blood $\mathrm{pH}$ changes after phosphate enema, water enema, and electrolyte lavage solution enema for flexible sig- moidoscopy. Gastrointest Endosc 1992;38:575-8. [ PubMed ]

21. Wendy S. Atkin, Andrew Hart, Robert Edwards, Clarie F. Cook, Jane Wardle, Peter Mclntyre et al. Single blind, 
randomised trial of efficacy and acceptibilty of oral picolax versus self adminstered phosphate enema in bowel prepration for flexible sigmoidoscopy screening. BMJ, 2000 Jun 3; 320(7248): 1504- 9.[ PubMed ]

22. Preston KL, Peluso FE, Goldner F. Optimal bowel preparation for flexible sigmoidoscopy - are two enemas better than one? Gastrointest Endosc 1994; 40: 474- 6. [ PubMed]
23. Sharma VK, Chockalingham SK, Ugheoke EA, Kapur A, Ling $\mathrm{PH}$, Vasudeva $\mathrm{R}$, et al. Prospective, randomized, controlled comparison of the use of polyethylene glycol electrolyte lavage solution in four-liter versus two-liter volumes and pretreatment with either magnesium citrate or bisacodyl for colonoscopy preparation. Gastrointest Endosc 1998 Feb; 47(2): 167-71.[ PubMed ] 